\title{
ARTICLE
}

\section{Propeller flaps in eyelid reconstruction}

\author{
Saul N. Rajak ${ }^{1,2} \cdot$ Shyamala C. Huilgol ${ }^{3} \cdot$ Masahiro Murakami $^{4} \cdot$ Dinesh Selva $^{2}$
}

Received: 30 November 2017 / Revised: 7 January 2018 / Accepted: 6 February 2018 / Published online: 14 March 2018

(c) The Royal College of Ophthalmologists 2018

\begin{abstract}
Purpose Propeller flaps are island flaps that reach the recipient site through an axial rotation. The flap has a subcutaneous pedicle on which it pivots, thereby resembling a helicopter propeller. We present our series of propeller flaps for the reconstruction of large eyelid defects.

Methods This is a retrospective review of the clinical case notes of eight patients that underwent tumour excision with reconstruction with a cutaneous propeller flap supplied by a non-perforator orbicularis pedicle between July and December 2016.

Results Propeller flaps were used in the reconstruction of five lower lid defects (size range $19 \times 5 \mathrm{~mm}$ to $25 \times 8 \mathrm{~mm}$ ), one medial canthus defect (13 mm diameter), one complete upper lid defect $(42 \times 19 \mathrm{~mm}$ diameter) and one lid sparing extenteration defect. The flaps were recruited from nasolabial, lateral canthal, temple or medial upper cheek skin. Post-operatively one case had 'trapdooring' which required flap revision at 4 months and one had persistent oedema that settled without intervention.

Conclusions The reconstruction of large eyelid defects is challenging in part because of the paucity of locally available skin. Propeller flaps are a paradigm shift in periocular reconstruction in which the subcutaneous pedicle enables the recruitment of large and highly mobile skin flaps from a wide area of regional tissue.
\end{abstract}

\section{Introduction}

Propeller flaps are defined as island flaps that reach the recipient site through an axial rotation [1,2]. The flap pivots on a subcutaneous pedicle, resembling a helicopter propeller. They are used in reconstructive surgery elsewhere in body, but rarely described for periocular reconstruction. We present our series of propeller flaps for the reconstruction of large eyelid defects.

Saul N. Rajak

Saul.rajak@bsuh.nhs.uk

1 The Sussex Eye Hospital, Brighton and Sussex University Hospital, Brighton, UK

2 South Australian Institute of Ophthalmology, Royal Adelaide Hospital, North Terrace, Adelaide, SA, Australia

3 Department of Dermatology, Royal Adelaide Hospital, University of Adelaide, Adelaide, SA 5000, Australia

4 Department of Plastic and Reconstructive Surgery, Nippon Medical School Musashi-Kosugi Hospital, Tokyo, Japan

\section{Methods and patients}

This is a retrospective review of the clinical case notes of consecutive patients at two institutions (Brighton and Sussex University Hospital, UK and The Royal Adelaide Hospital, Australia) that underwent tumour excision with reconstruction with a cutaneous propeller flap supplied by a random (non-perforator) orbicularis pedicle between July and December 2016. Informed consent was given by all patients for the procedure and clinical photographs.

\section{Surgical technique}

If the propeller flap is being used for eyelid reconstruction, the posterior lamella can be reconstructed with a graft, for example a free tarsal graft harvested from the upper eyelid (Fig. 1a). Elsewhere in the periocular area two-layer reconstruction is not required and the propeller flap alone can be used.

The flap is designed to recruit tissue from an area of sufficient laxity with an appropriate skin match and preferably siting scars along relaxed skin tension lines or on borders of cosmetic subunits. The size of the flap is templated from the defect, aiming to create the minimum sized 
Fig. 1 Propeller flap technique. a Large left lower eyelid defect. b (i) Posterior lamella reconstructed with free tarsal graft harvested from upper eyelid. (ii) Propeller flap marked: • Location: along relaxed skin tension lines or borders of cosmetic subunits in area with sufficient skin laxity and to mimimise rotation. $\cdot$ Size: templated from defect to minimum possible size/slightly undersized. • Pedicle location: one-quarter of diameter of flap from proximal edge. c Flap gradually raised around full circumference towards planned pedicle site. Pedicle initially kept wide and then gradually thinned and lengthened until the flap rotates freely into defect without tension. d Flap transposed into defect to reconstruct anterior lamella. e Primary closure of secondary defect

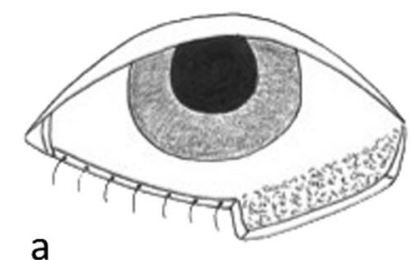

a

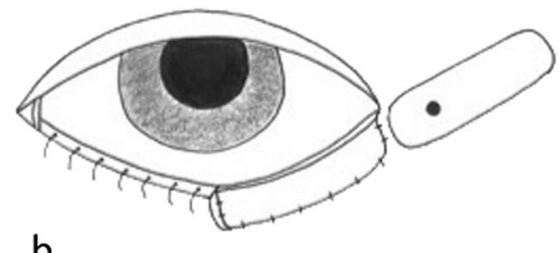

b
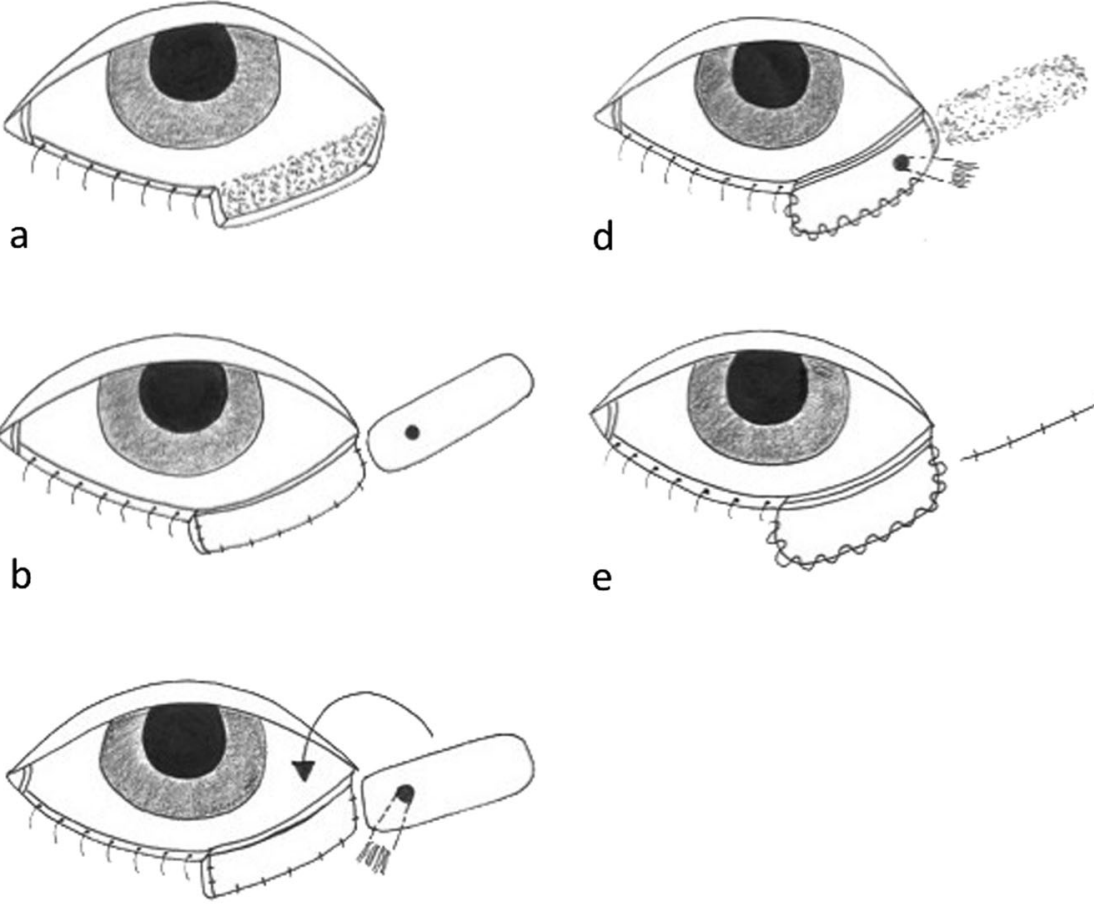

C

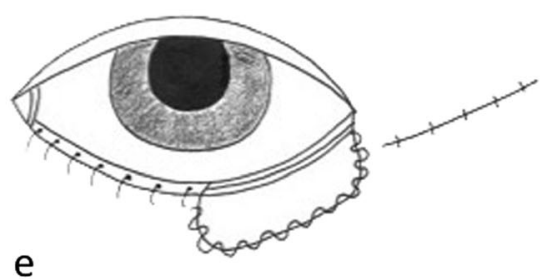

flap required, or possibly even to undersize it slightly, which may reduce the incidence of post-operative trapdooring or oedema. The propeller flap is positioned to minimise the amount of rotation required and has one point adjoining the defect (Fig. 1b).

The location of the pedicle is determined by the site of the defect and the positioning of the flap, but is typically one-quarter of the flap diameter away from the defect. In general, an inferior pedicle is preferred, to enhance lymphatic drainage and possibly reduce the risk of postoperative oedema and trapdooring. The flap and pedicle positon may also be affected by whether an orbicularis or subcutaneous pedicle is preferred.

The full circumference of the flap is incised and the flap is dissected free in either the subcutaneous or suborbicularis plane as per the desired flap thickness (Fig. 1c). The flap can be thinned, but not excessively in order to preserve the subdermal plexus, maintaining flap vascularity. As the pedicle area is approached dissection is in the suborbicularis plane and an orbicularis pedicle is fashioned although a shorter subcutaneous pedicle can also be created. A wide pedicle is created initially and it is gradually reduced whilst checking the rotation after each reduction, until a pedicle is created with the maximum diameter that will will allow adequate rotation without excessive torque. The pedicle length can be increased by dissecting out more orbicularis, but should be the minimum length required to allow the flap to reach the defect with tension on the pedicle. Typically, a pedicle of diameter $6-10 \mathrm{~mm}$ and length $10-14 \mathrm{~mm}$ is created. The flap is rotated and transposed into the defect, taking care not to accidentally rotate the pedicle any more than required (Fig. 1d). The flap can be rotated and transposed slowly or in stages to maintain perfusion; the donor site can be sutured during this time. The flap is sutured into position and the placement of tacking sutures to secure the base of the flap is advisable (Fig. 1e).

\section{Results}

Propeller flaps were used in the reconstruction of eight periocular tumour excision cases. Seven of the patients were male and the average age was 74 . Four lower lid defects (size range $19 \times 5 \mathrm{~mm}$ to $25 \times 8 \mathrm{~mm}$ ) were reconstructed with medial cheek propeller flaps rotated around $90^{\circ}$ as seen in Fig. 2 and one lower lid defect was reconstructed with a lateral canthal propeller flap rotated $180^{\circ}$ (similar to Fig. 3). One medial canthus defect (13 $\mathrm{mm}$ diameter) was reconstructed with a medial cheek propeller flap transposed over a small bridge of healthy tissue with an incision in it to accommodate the subcutaneous orbicularis pedicle (Fig. 4). The anterior lamellar of one complete upper lid defect $(42 \times 19 \mathrm{~mm}$ diameter, Fig. 5) was reconstructed with a propeller flap recruited from the temple. A partial lid sparing exenteration defect was reconstructed with a lateral canthal propeller flap (Fig. 3). In all cases, the pedicle was sited 
Fig. 2 Propeller flap reconstruction of the lower eyelid. a Nasolabial propeller flap marked, b posterior lamella reconstructed with free tarsal graft from contralateral upper lid, c propeller flap harvested with orbicularis pedicle, $\mathbf{d}$ propeller flap rotated $90^{\circ}$ and sutured in situ and harvest site closed by primary intention

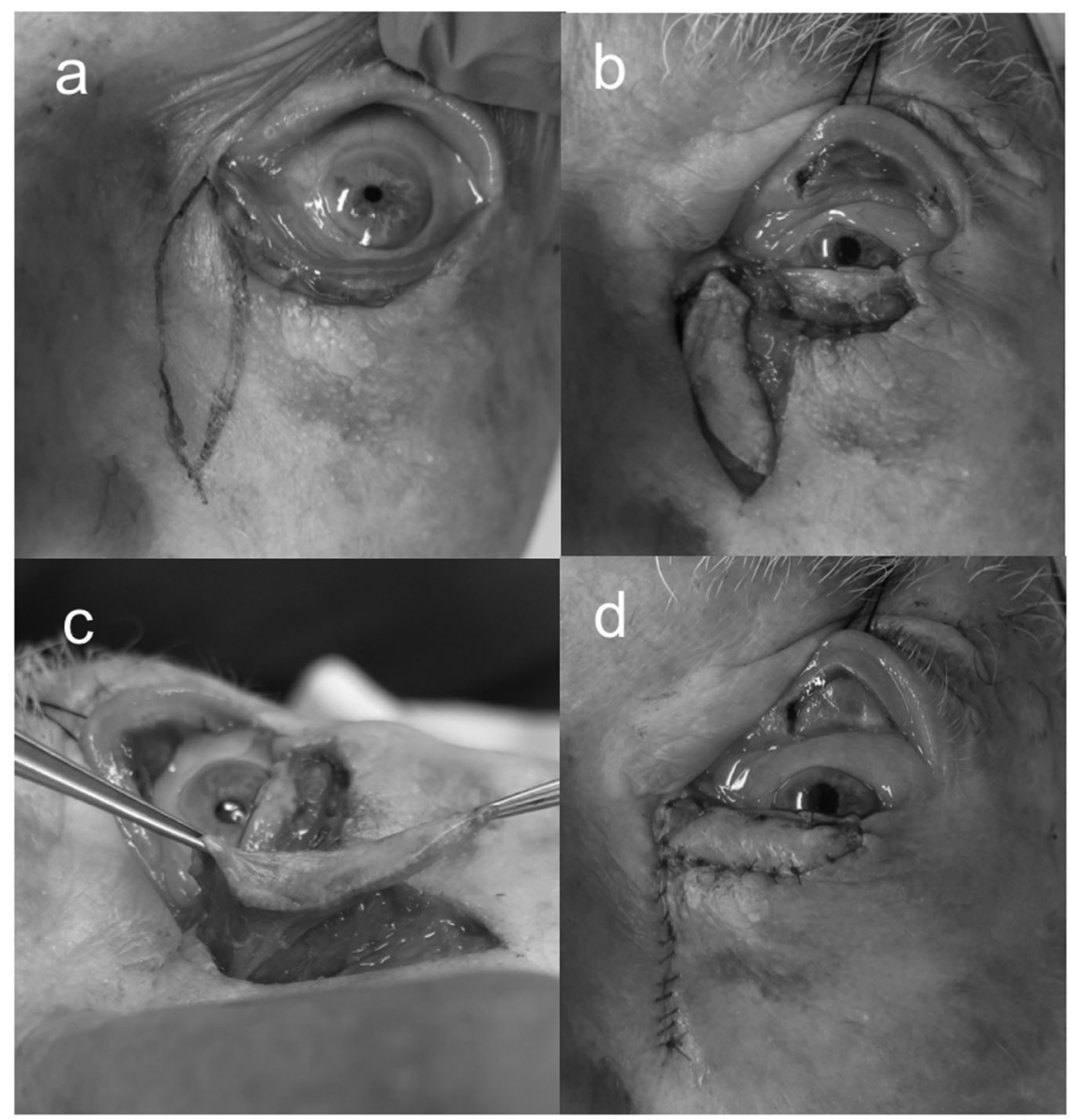

one-quarter of the flap diameter away from the defect. There were no intra-operative complications and clear margins were achieved in all cases. Post-operatively, healed well without any areas of necrosis. The lower lid reconstructions resulted in excellent cosmetic and functional outcomes except for one patient who had flap 'trapdooring' that required flap revision 4 months postoperatively and one patient who had persistent lower eyelid oedema that settled without intervention 4 months post-operatively. The upper eyelid reconstruction participant had mild but untroublesome upper eyelid retraction (see Fig. 4). All patients have been followed up for 6-12 months.

\section{Discussion}

Propeller flaps are widely used in plastic and reconstructive surgery but have rarely been described for reconstruction of the periocular area, where they are particularly advantageous for larger reconstructions in view of the paucity of immediately neighbouring skin.

The term propeller flap was original used to denote a local island fasciocutaneous flap based on a single dissected perforator, while flaps with random subcutaneous pedicle have been called subcutaneous island flaps [3]. However, a wider definition of propeller flaps was agreed at the first Tokyo meeting on perforator and propeller flaps, with subclassification by pedicle type [1]: random [2], skeletonised perforator vessel and [3] 'supercharged' in which an anastomosis is also created to increase arterial inflow or venous outflow $[1,4]$.

The propeller flap can be fashioned with a long orbicularis pedicle. This enables relatively large flaps to be transposed over a greater distance than skin pedicle flaps and without distortion at the base of the flap. This is particularly advantageous in the periocular area where there is a shortage of immediately neighbouring skin but there is redundancy in nearby areas such as the temple and perinasal area. The nasojugal propeller flaps have similar utility and aesthetic outcomes to conventional skin-based nasojugal flaps, although the pedicle of the propeller flap could be used to bridge a small area of the healthy skin.

Conventional plastic surgical teaching generally advises that random (non-propeller) skin flaps should not have a length of more than four times their diameter, although this varies according to tissue vascularity. The present series 
Fig. 3 Propeller flap reconstruction of a lid sparing exenteration defect. a Temporal/ zygomatic flap marked, b flap raised with pedicle of maximum possible thickness that allows rotation, $\mathbf{c} 180^{\circ}$ rotation and suturing into defect and repair of secondary defect, $\mathbf{d}$ appearance 8 days post-operation

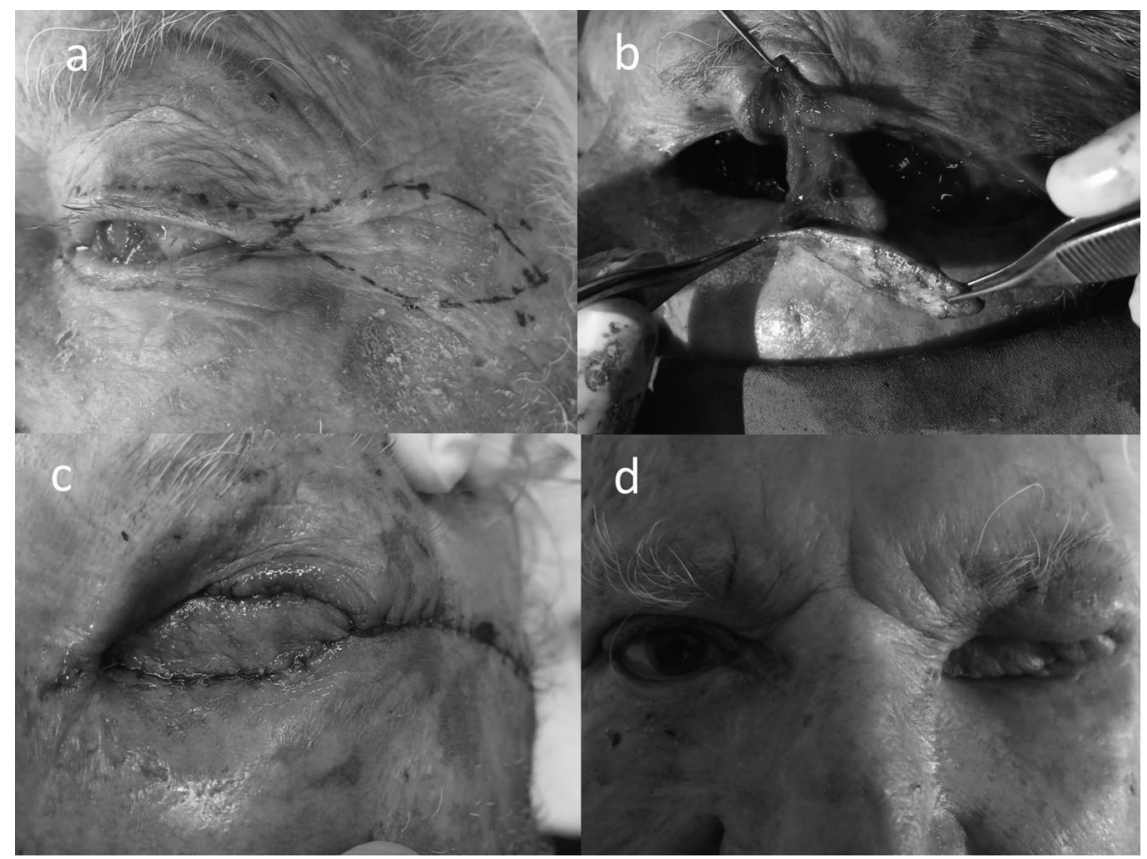

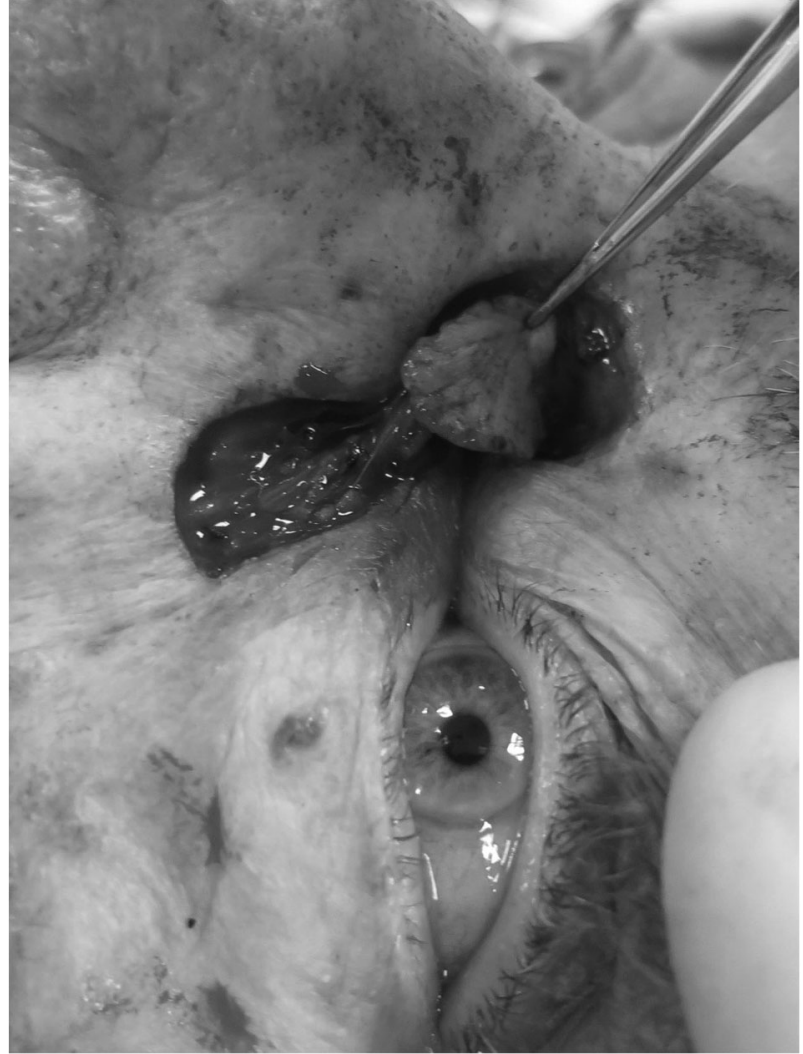

Fig. 4 Medial canthal reconstruction using a medial cheek propeller flap. The flap is being transposed over a small bridge of normal skin with a cut in it to accommodate the subcutaneous orbicularis pedicle

shows that greater ratios can be tolerated in the highly vascular periocular area with large flaps being supported by relatively small random orbicularis pedicles in the reconstruction of an exenteration defect and the anterior lamella of a large upper lid defect (patients 7 and 8). Interestingly, patient 7 reached near critical ischaemia in the first two post-operative days, before the flap fully perfused without complication. However, as this patient had advanced microvascular disease, diabetes and was a smoker, even greater flap dimension to pedicle size may be achievable with healthy vasculature.

Random pedicle propeller flaps are generally used for rotations of up to $90^{\circ}$. However, the present series reaffirms two previous reports that in the highly vascular periocular area, $180^{\circ}$-rotations are effective [5, 6]. It is generally recommended that once the flap has been fully prepared ('islanded'), it is left in situ for $10-15 \mathrm{~min}$ to allow it to fully perfuse prior to rotating the pedicle and potentially reducing perfusion. Other parts of the procedure such as secondary defect repair can be conducted during this period to avoid increasing surgical time.

The trapdoor effect is the elevation or bulging of tissues within the boundaries of scar [7]. It probably derives predominantly from lymphatic and venous outflow impedance, although scar hypertrophy and redundant subcutaneous fatty tissue may also contribute to its development. It typically occurs around 3 weeks after surgery but can develop as late as 6-8 months post-operatively. It occurs more commonly in island flaps than skin-based flaps perhaps because they need to be thicker to maintain a vascular plexus. The one case in which this occurred in the present series was a nasolabial/medial cheek flap. This location may be particularly prone to this complication, perhaps because the pedicle is by necessity superior. Propeller flaps may be 


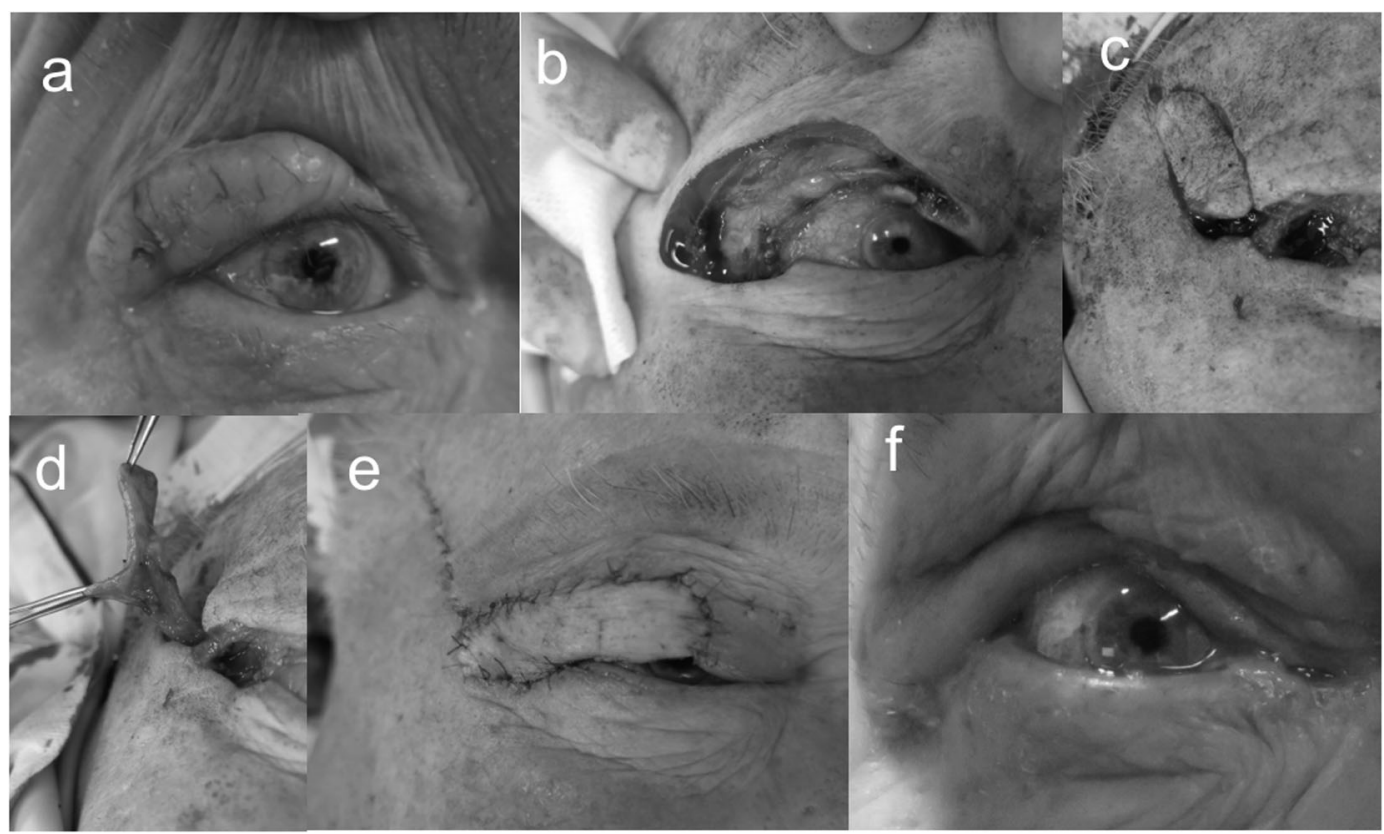

Fig. 5 Upper eyelid reconstruction using a propeller anterior lamellar flap to support a free tarsal graft from the contralateral upper eyelid. a Upper eyelid nodular basal cell carcinoma, $\mathbf{b}$ defect after excision with $3 \mathrm{~mm}$ margins, c and d propeller flap harvested with random

at particular risk of the trapdoor effect, because of the small diameter pedicle relative to the size of the flap. Various surgical strategies have been suggested for minimising trapdooring, such as tacking the flap base down to the host bed, avoiding oversizing of the flap (or even undersizing by templating the true defect size rather than the relaxed postexcision defect), ensuring the flap has straight edges and geometric angles, undermining widely beyond the base of the flap and depressing or insetting the flap slightly within the defect as it will rise in time. However, insetting is not possible in the thin skin of the eyelid. Most cases settle with time and/or steroid injections and revision surgery is infrequently required.

The vascular supply to the orbicularis oculi muscle comes from small vertical branches that enter the deep side of the muscle from the vascular arcades and plexus of anastomoses in the periocular area [8]. Therefore, in theory propeller flaps may be best supported by leaving orbicularis muscle, as this may improve blood supply to the more distal aspects of the flap. However, in practice, we have used both subdermal and suborbicularis flaps in this series without clear evidence of a difference in outcome.

There are several other reports of periocular propeller flaps. Baltu et al. describe a glabellar propeller flap based on a central artery perforator artery for the reconstruction of nasal and medial canthal defects [9]. This increases the mobility and flexibility of the traditional glabellar flap but leaves the prominent central scar. Despite the use of a orbicularis pedicle approximately one-quarter of the length of the flap from its proximal end, e flap sutured in situ and harvest site defect primarily closed, f 3 months post-operative appearance

named perforator, they still report $5 / 22$ cases with venous congestion of which two had partial flap loss and the present series suggests that random flaps which open up a much wider area of potential harvest sites are equally successful. Large lower eyelid defects have been reconstructed with non-perforator (random) pedicle lateral canthal propeller flaps rotated $180^{\circ}$. However, in contrast to the present series, in two reports totalling 14 cases, the graft is used to support posterior lamella palatal mucosal graft (rather than a free tarsal graft) and in another report of five cases, the origin of the flap, size of defect and posterior lamella tissue are not described $[5,6,10]$. The reconstruction of medial canthal defects with a propeller flap has also been described, but is referred to as an island pedicle advancement flap [11]. The technique described is similar to the present case of medial canthal reconstruction, except that Lee et al. describe the flap being harvested with a long common border with the defect, when in fact the propeller flap allows for skin to be recruited from an area that is only just adjoining the defect or transposed over a small bridge of healthy tissue with an incision made in the bridge to allow the pedicle running subcutaneously. There are no previous reports of propeller flaps being used to reconstruct upper lid or exenteration defects, where they have particular benefits. In the upper eyelid, it allowed a one-stage procedure in an anaesthetically high-risk patient who could not tolerate a local anaesthetic, rather than the two-stage procedures 
such as the modified Cutler Beard that are typically used because of the paucity of locally available skin to support a posterior lamella graft. For the partial lid sparing exenteration, the propeller flap comfortably filled the defect with a much shorter and less invasive operation than a temporalis or a paramedian forehead flap.

Propeller flaps are a paradigm shift in periocular reconstruction in which the subcutaneous pedicle enables the recruitment of large and highly mobile skin flaps from a wide area of regional tissue.

\section{Summary}

\section{What was known before:}

- Eyelid reconstruction can be challenging because of the paucity of locally available tissues. Skin flaps are invariable based on a skin pedicle which limits their mobility.

\section{What this study adds:}

- Propeller flaps facilitate recruitment of a much wider area of skin for periocular reconstruction and can support posterior lamella grafts

\section{Compliance with ethical standards}

Conflict of interest The authors declare that they have no conflict of interest.

\section{References}

1. Pignatti M, Ogawa R, Hallock GG, Mateev M, Georgescu AV, Balakrishnan G, et al. The "Tokyo" consensus on propeller flaps. Plast Reconstr Surg. 2011;127:716-22.

2. Hyakusoku H, Yamamoto T, Fumiiri M. The propeller flap method. Br J Plast Surg. 1991;44:53-4.

3. Teo TC. The propeller flap concept. Clin Plast Surg. 2010;37:615-26. vi

4. D'Arpa S, Toia F, Pirrello R, Moschella F, Cordova A. Propeller flaps: a review of indications, technique, and results. Biomed Res Int. 2014;2014:986829.

5. Miyamoto J, Nakajima T, Nagasao T, Konno E, Okabe K, Tanaka $\mathrm{T}$, et al. Full-thickness reconstruction of the eyelid with rotation flap based on orbicularis oculi muscle and palatal mucosal graft: long-term results in 12 cases. J Plast Reconstr Aesthet Surg. 2009;62:1389-94.

6. Nakajima T, Yoshimura Y. One-stage reconstruction of fullthickness lower eyelid defects using a subcutaneous pedicle flap lined by a palatal mucosal graft. $\mathrm{Br}$ J Plast Surg. 1996;49:183-6.

7. Koranda FC, Webster RC. Trapdoor effect in nasolabial flaps. Causes and corrections. Arch Otolaryngol. 1985;111:421-4.

8. Lopez R, Lauwers F, Paoli JR, Boutault F, Guitard J. The vascular system of the upper eyelid. Anatomical study and clinical interest. Surg Radiol Anat. 2008;30:265-9.

9. Baltu Y, Uzun H, Dolen UC, Ozyurtlu M. Central artery perforator propeller flap for nasal and medial canthal defects. J Plast Reconstr Aesthet Surg. 2016;69:e180-5.

10. Kim YJ, Han JK, Lee SI. A subcutaneous pedicled propeller flap: new alternative technique for lower eyelid reconstruction after excision of basal cell carcinoma. J Plast Reconstr Aesthet Surg. 2012;65:1434-6.

11. Lee BJ, Elner SG, Douglas RS, Elner VM. Island pedicle and horizontal advancement cheek flaps for medial canthal reconstruction. Ophthal Plast Reconstr Surg. 2011;27:376-9. 